\title{
Non-covalent interactions between carbon nanotubes and conjugated polymers
}

\author{
Dönüs Tuncel* \\ Received 30th March 2011, Accepted 14th June 2011 \\ DOI: $10.1039 / \mathrm{c} 1 \mathrm{nr} 10338 \mathrm{e}$
}

\begin{abstract}
Carbon nanotubes (CNTs) are interest to many different disciplines including chemistry, physics, biology, material science and engineering because of their unique properties and potential applications in various areas spanning from optoelectronics to biotechnology. However, one of the drawbacks associated with these materials is their insolubility which limits their wide accessibility for many applications. Various approaches have been adopted to circumvent this problem including modification of carbon nanotube surfaces by non-covalent and covalent attachments of solubilizing groups. Covalent approach modification may alter the intrinsic properties of carbon nanotubes and, in turn make them undesirable for many applications. On the other hand, a non-covalent approach helps to improve the solubility of CNTs while preserving their intrinsic properties. Among many noncovalent modifiers of CNTs, conjugated polymers are receiving increasing attention and highly appealing because of a number of reasons. To this end, the aim of this feature article is to review the recent results on the conjugated polymer-based non-covalent functionalization of CNTs with an emphasis on the effect of conjugated polymers in the dispersibility/solubility, optical, thermal and mechanical properties of carbon nanotubes as well as their usage in the purification and isolation of a specific single-walled nanotube from the mixture of the various tubes.
\end{abstract}

\section{Introduction}

Ever-increasing demand for materials with defined functions and properties urge researchers to seek for ways to design and synthesize novel materials. To this end, nanostructured-materials are getting increasing attention owing to their interesting and unusual properties. Among them, the carbon nanotubes (CNTs) are of particular interest to researchers from different disciplines

Deparment of Chemistry and Institute of Materials Science and Nanotechnology, Bilkent University, Bilkent, Ankara, Turkey 06800. E-mail: dtuncel@fen.bilkent.edu.tr such as chemistry, physics, biology, medicine and engineering because of their extraordinary structural, mechanical and electronic properties and also for their potential applications in many areas including optoelectronics, nanotechnology, nanomedicine, chemical and biological sensing and etc. ${ }^{1-8}$

CNTs having a cylindrical nanostuctured-shape consist of seamless rolls of graphitic sheets and can be classified as singlewalled nanotubes (SWCNTs) and multi-walled carbon nanotubes (MWCNTs) depending on the number of graphene sheets they contain as shown in Fig. 1. Diameter of SWCNTs could vary between $0.4 \mathrm{~nm}$ and $3 \mathrm{~nm}$ while a diameter range of 2-30 nm is characteristic for MWNTs. The length of both SWCNTs and

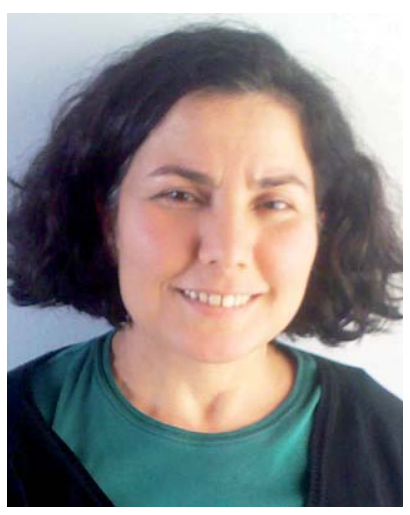

Dönüs Tuncel
Dönüs Tuncel graduated from University of North London. Then she went on to study her PhD degree at Cambridge University in the Melville Polymer Synthesis Laboratory and after a while she moved to Imperial College, London and completed her study in 1999 under the supervision of Dr Joachim Steinke in the area of supramolecular chemistry of cucurbiturils. She worked for about 3 years as a postdoctoral research assistant in the Chemistry Department of Oxford University under the direction of Professor Harry L. Anderson. Since 2003, she has been working in the Department of Chemistry at Bilkent University as an Assistant Professor. D. Tuncel's research is highly interdisciplinary and combines the synthetic organic, supramolecular and polymer chemistry to prepare functional materials such as supramolecular polymers, cucurbituril containing rotaxanes and poyrotaxanes, water soluble conjugated polymers, functional and mechanically-stable multi-functional conjugated polymer nanoparticles which have potential applications in the use of polymeric opto-electronic devices (LEDs, solid state lighting and photovoltaic devices), molecular switches as well as biomedicine. 


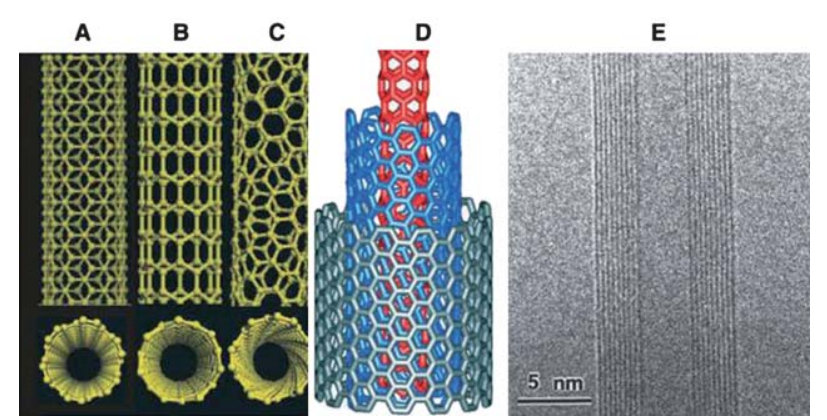

Fig. 1 Schematic illustrations of the structures of (A) armchair, (B) zigzag, and (C) chiral SWCNTs (D) MWCNT (E) Transmission electron microscope (TEM) image of a MWNT containing a concentrically nested array of nine SWNTs (adapted with permission from ref. 4. Copyright 2002 by the American Association for the Advancement of Science).

MWCNTs are usually microns to millimetres long. The orientation of the hexagon rings along the tubular surface determines whether CNTs possess metallic or semiconducting character and a mixture of SWCNTs contains both metallic and semiconducting nanotubes with varying sizes. ${ }^{1-4}$

Although CNTs possess many unique properties and hold promise for many applications, their usage is rather limited and in order to expand their applications some of the problems should be addressed. For instance, one of the drawbacks associated with these materials is their well-known intractability that they are insoluble in any solvents and infusible at any temperatures before decomposing. Their insolubility stems from the bundling of individual carbon nanotubes caused by the summation of van der Waals interactions between the walls of nanotubes. These bundles can be quite large and, thereby, it can be difficult to disperse them in either aqueous or non-aqueous media. To this end, many approaches have been pursued to circumvent this problem including modifying the walls of CNTs through a covalent functionalization, ${ }^{9-11}$ and dispersants-assisted dispersion via non-covalent interaction. ${ }^{12-14}$

In the covalent functionalization, CNTs are treated with strong oxidizing reagents to form functional groups on the nanotube walls and these functional groups are further reacted with other functionalities that can make CNTs water or organic solvent soluble depending on the hydrophilicity/hydrophobicity of the species attached to the nanotubes. Although this method ensures good solubility of CNTs in various solvents, it usually alters the intrinsic properties of CNTs such as conductivity, mechanical strength and optical properties.

In the non-covalent functionalization, CNTs are added to a dispersant containing solution and the mixture is agitated through sonication. In this way, the CNTs are first mechanically de-bundled and then stabilized by dispersant molecules through non-covalent interactions by causing no substantial changes in the chemical structures, electronic and mechanical properties of the carbon nanotubes. Therefore, this method is quite appealing for many applications.

Surfactants, biomolecules and polymers are widely used as dispersants and non-covalent modifiers of CNTs. ${ }^{15-19}$ Among them the polymers are quite efficient dispersants because of their long chain structure that can wrap themselves around CNTs by disrupting the van der Waals interactions between the walls of
CNTs. Conjugated polymer-based functionalization of CNTs are particularly interesting because of a number of reasons. ${ }^{19-66}$ First, the solubility and consequently processability of CNTs could be improved by using conjugated polymers (CPs) as dispersant agents because of their extensive pi-conjugated backbone that can interact with the side-walls of CNTs through $\pi-\pi$ interactions. Second, CNT/CP nanocomposites could be obtained that have been reported to exhibit certain synergetic effects arising from the good thermal-/photostability, high mechanical strength, and high electrical conductivity of carbon nanotubes as well as excellent optoelectronic properties of conjugated polymers. The applications of these composite materials have been demonstrated in the fabrication of chemical sensors, photovoltaic devices, field effect transistors and transparent electrodes. ${ }^{20-32}$ Third, CPs can be used in the purification and extraction of a specific SWNT from a mixture of the various tubes. Finally, luminescent properties of CPs can be real advantages for some applications such as biological imaging and sensing if CNTs are dispersible in water-soluble conjugated polymers.

Accordingly, this feature article will discuss the recent results on the conjugated polymer-based non-covalent functionalization of CNTs with an emphasis on the effect of conjugated polymers in the dispersibility/solubility, optical, thermal and mechanical properties of carbon nanotubes as well as their usage in the purification and isolation of a specific SWNT from the mixture of the various tubes.

\section{Conjugated polymers used as a dispersant agent in organic solvents}

Conjugated polymers (CPs) have been found to disperse CNTs very efficiently in organic solvents. This is a quite important feature in the preparation of films and nanocomposites of CPs/ CNTs. These materials combine the good thermal-/photostability, high mechanical strength, and high electrical conductivity of carbon nanotubes with excellent optoelectronic properties of conjugated polymers and consequently can find many applications in device fabrications including light emitting diodes, solar cells, transparent conductive films and sensors.

The studies show that the conjugated polymers assist the dispersion of CNTs in solvents by forming supramolecular complexes through either by helical wrapping or non-helical adsorption but through $\pi$-stacking depending on the flexibility of the polymer backbone. There are some mechanistic and computational studies in the literature to reveal the exact nature of these interactions; however, they are rather limited. In both cases, the CNTs are agitated through stirring or ultrasonication in the solution of CPs in an organic solvent or in water. CNTs are de-bundled and individual tubes interact with CPs to form stable complexes which do not go re-bundling indefinitely. This section will review the recent studies involving the use of conjugated polymers and conjugated block copolymers as a dispersant agent in organic solvents.

Poly(phenylenevinylene) derivatives such as P1 (Fig. 2) have been extensively studied for use as a polymeric dispersion agent for CNTs. Molecular mechanics energy minimization calculations indicate that the polymer chain tends to form a helical structure due to the dihedral angle caused by the meta-phenylene 


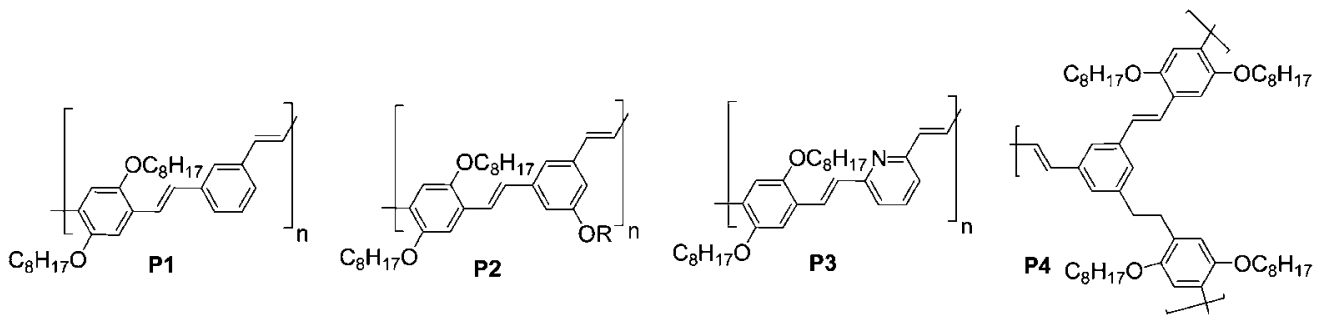

Fig. 2 Structures of poly(m-phenylene-co-2,5-dioctoxy-p-phenylenevinylene) (P1), poly(5-alkoxy-m-phenylenevinylene)-co-(2,5-dioctoxy-p-phenylene)-vinylene (P2), poly(2,6-pyridinylenevinylene)-co-(2,5-dioctyloxy-p-phenylene)vinylene (P3) and stilbene-like dendrimer (P4).

linkages in the polymer backbone. P1 interacts with the sidewalls of carbon nanotubes and form stable dispersions of complexes in organic solvents indefinitely by wrapping itself around the CNTs as the polymer structure possesses helicity. It was also observed that the composite materials of CNTs with P1 have exhibited nearly eightfold increases in electrical conductivity $\left(10^{-2} \mathrm{~S} / \mathrm{m}\right)$ compared with pristine P1 $\left(10^{-10} \mathrm{~S} / \mathrm{m}\right)$. Moreover, the photoand/or electroluminescence properties of the polymer were not altered upon composite formation. Application of the composite $\mathbf{P 1 / C N T s}$ has been demonstrated in electrical conducting films and optoelectronic devices. ${ }^{20,28-32}$

Stoddart and co-workers investigated the non-covalent interactions of a series of conjugated polymers including P1, P2, P3, and stilbene-like dendrimer (P4) (the chemical structures of these polymers are shown in Fig. 2) with CNTs. ${ }^{34-36}$ They used an excess of $\mathbf{P 1}$ to solubilize SWNTs in chloroform and found that the length and diameter distribution of the SWCNT ropes was dependent on the concentrations of P1 and SWNTs. It was shown by optical imaging and optoelectronic measurements on single, isolated P1-wrapped SWNT ropes that polymer-wrapped SWNTs have not further aggregated into thicker ropes, and that the polymer and nanotube components of a wrapped structure are in intimate electrical contact with each other. Moreover, the authors have performed a computer modelling studies by molecular mechanics and molecular dynamics simulations to reveal the nature of the interaction between a short-SWNT and a $\mathbf{P 1}$ of eight repeating units by confirming that the polymer is wrapped helically around the nanotubes with the phenylene rings in the polymer backbone stacked in a face-to-face manner against the walls of the nanotube. ${ }^{33}$

The same group further investigated the interactions between SWNTs and P3. Since $\mathbf{P} 3$ contains a pyridine unit that acts as a base, it is readily protonated via the addition of $\mathrm{HCl}$. They found that the SWNT/P3 interaction lowers the $\mathrm{p} K_{\mathrm{a}}$ of $\mathbf{P 3}$. Optoelectronic devices, fabricated from single polymer-wrapped SWNT structures, reveal a photogating effect on charge transport which can rectify or amplify current flow through the tubes. For $\mathbf{P 1}$ wrapped tubes, the wavelength dependence of this effect correlates to the absorption spectrum of P1. For P3, the wavelength dependence correlates with the absorption spectrum of protonated P3, indicating that SWNTs assist in charge stabilization. ${ }^{34}$

It was shown using spectroscopic techniques such as UV-Vis absorption and Raman as well as electron microscopy that the purification of CNTs from carbonaceous impurities was possible using $\mathbf{P 1}$ as an extracting agent. The solutions of $\mathbf{P 1}$ selectively dispersed CNTs well leaving behind the impurities as undissolved particles. ${ }^{30}$ In another work, P1's ability to form a helical conformation was found to be important in the separation of nanotubes. P1 was found to selectively interact with the tubes having the diameters of $1.19,1.25$ and $1.24 \mathrm{~nm}$, respectively, among about 15 tubes present in the pure SWNT sample. The SWNTs of smaller diameters were held loosely by the $\mathbf{P 1}$, and were gradually precipitated during centrifugation. The suspension prepared from the SWNT and P1 was observed to be not permanently stable; precipitation occurs after a few weeks. Furthermore, irradiation in the UV-vis region exhibited a catalytic effect to shorten the precipitation time to hours. ${ }^{37}$

Two oligo(phenylenevinylene)s (P5 and P6) terminated with cyanobiphenyl (Fig. 3) were synthesized as non-covalent modifiers of SWNTs. The wrapping of SWNTs with these oligomers enhanced the dispersibility in $\mathrm{CHCl}_{3}$ by the dissociation of SWNT bundle structures. It was found that the addition of oligomers causes self-organized precipitation from a homogeneous dispersed solution of SWNT complexes. The self-organized precipitates exhibit liquid-crystalline properties. ${ }^{38}$

A detailed study conducted by Pang and co-workers using UV-Vis absorption and fluorescence spectroscopy showed that the poly[(m-phenylenevinylene)-alt-(p-phenylenevinylene) $]$ with cis-vinylene bonds is a more efficient dispersant for SWNTs than its trans isomer. It was proposed that the chain conformation of the $c i s-\mathrm{CH}=\mathrm{CH}$ bond geometry, formed a suitable cavity among chain segments to wrap the tubes through the interaction of a planar conjugated polymer backbone with the SWNT. The cisisomer-wrapped SWNTs were found to have good solubility and also observed to be not selective in the dispersion of various

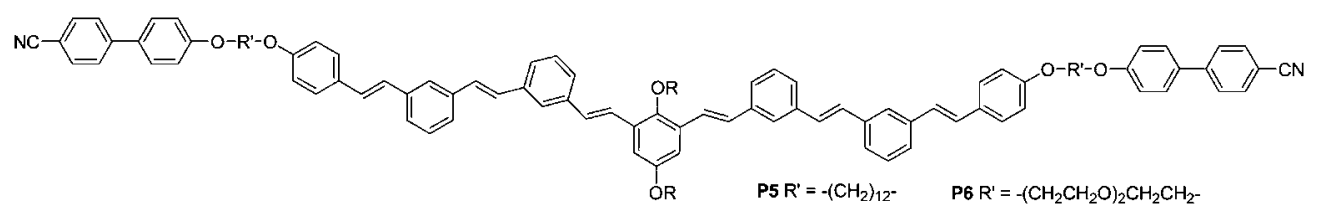

Fig. 3 The structures of two oligo(phenylenevinylene)s terminated with cyanobiphenyl were used as non-covalent modifiers of SWNTs. 
kinds of SWNTs in the sample, in contrast to its trans-isomer which selectively interacted with the metallic SWCNTs. ${ }^{39}$

Tang and co-workers synthesized a group of pyrene-containing poly(phenylacetylene)s (P7, P8 and P9) (Fig. 4) to study their interactions with CNTs in organic solvents. MWCNTs were efficiently dispersed in the chloroform or THF solution of these polymers with a maximum MWCNT concentration of about $0.64 \mathrm{mg} / \mathrm{mL}$. They attributed this to the spontaneous wrapping of the polymer chains themselves around the MWNTs due to the favorable $\pi-\pi$ interactions of the polymer backbone and the pyrene rings with the MWNT walls. The thermal properties of the composite of P7(5) with MWNT were studied and found that the composite was thermally stable and lost only some of its weight when heated to $338{ }^{\circ} \mathrm{C}$. The authors claimed that upon interaction of PPA with MWCNT, the conjugation length of the backbone became longer and when the composite material was excited it emitted blue-green light more efficiently than the polymer itself. The photovoltaic cell fabricated from the P2(5)/ MWNT composite found to be bipolar indicating an efficient photoinduced charge transfer takes place between P2(5) and MWNT. ${ }^{40}$

After using conjugated polymers with flexible backbone that can form helical structure to wrap around CNTs to make them dispersible in solvents, first time Chen and co-workers demonstrated that poly(phenylenevinylene)s (P10) (Fig. 5) having a rigid backbone could also be used to disperse SWNTs in various solvents and the maximum dispersion was observed to be $2.2 \mathrm{mg} \mathrm{mL} \mathrm{m}^{-1}$ in chloroform. $\mathbf{P 1 0}$ cannot wrap around the SWNTs owing to its rigid backbone, the major interaction between polymer backbone and nanotube surface is most likely through $\pi$-stacking. The strength of $\pi-\pi$ interactions between the SWNT surface and the polymer determine the efficiency of dispersion as well as stabilization. They investigated the effect of polymer rigidity on the solubility of SWNTs, by varying the backbone lengths. P10 polymers with backbone lengths less than $15 \mathrm{~nm}$ make the SWNTs highly soluble, providing further evidence for an interaction that does not involve polymer

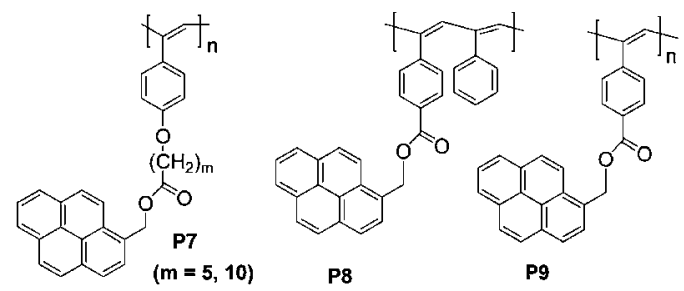

Fig. 4 The structures of a group of pyrene-containing poly(phenylacetylene)s (P7, P8 and P9). wrapping. The authors characterized the complex of P10/CNTs by ${ }^{1} \mathrm{H}-\mathrm{NMR}$, UV-Vis, Fluorescent spectroscopies and transmission electron microscopy (TEM) by confirming $\pi-\pi$ interaction between CNTs and P10. ${ }^{41,42}$

In 2006 four representative linear conjugated poly(phenylenevinylene)s (P10, P11, P12 and P13) (Fig. 5) were synthesized to further reveal the relationship between structurally rigid nonhelical conjugated polymers and various CNTs. Long n-decyloxy chains were grafted on the rigid backbones of these polymers in order to impart solubility in organic solvents as well as to stabilize SWNT dispersions by steric repulsion. Dispersibility tests of CNTs in chloroform solutions of polymers showed that $\mathbf{P 1 0}$ and P11 assisted the dispersion less efficiently than polymers with longer $\pi$-extended repeating units, P12 and P13 that both $\mathbf{P 1 2}$ and P13 could result in very stable dark-coloured solutions lasting for months without precipitation with the as-prepared SWNT powders after a short period of bath sonication (less than $5 \mathrm{~min}$ ). Good quality films were formed after evaporation of the solvent and these films could be easily redissolved in chloroform to form homogeneous solutions by sonication. Rheological studies showed that the dynamic viscosities of the SWNT dispersions increased compared with the corresponding polymer solutions. The results indicated that the polymers P12 and P13 can effectively interact with the as-prepared SWNTs to form stable supramolecular assemblies in chloroform. The strong interactions between SWNTs with polymers P12 and P13 were also supported by fluorescence analysis. The emission intensities of P12 and P13 were found to be considerably quenched after complexing with as-prepared SWNTs due to photoinduced charge transfer from polymers (donor) and SWNTs (acceptors) ${ }^{43}$ indicating that these composite materials could be used in the photovoltaic and photoconducting device fabrications.

Although fluorene based conjugated polymers possess rigid backbone with extended conjugation required for $\pi$-stacking to the nanotube surface to act as a dispersant, they have attracted rather limited attention. Recently, fluorene-based conjugated polymers (P14) and (P15) were synthesized and their complexes with SWCNTs were prepared (Fig. 6). P14 and P15 were found to form strong supramolecular complexes through non-covalent interactions with SWNTs, imparting good solubility in organic solvents in the absence of excess free polymer. Thermogravimetric analysis indicated that the polymer nanotube complexes were composed of $40 \mathrm{wt} \%$ polymer (Fig. 7). UV-vis absorption measurements revealed a bathochromic shift in the absorption spectrum of the polymers as a result of nanotube complexation, while fluorescence measurements showed that polymer fluorescence was highly quenched in the complexes. Microscopic techniques (atomic force microscopy (AFM), and TEM) were used to
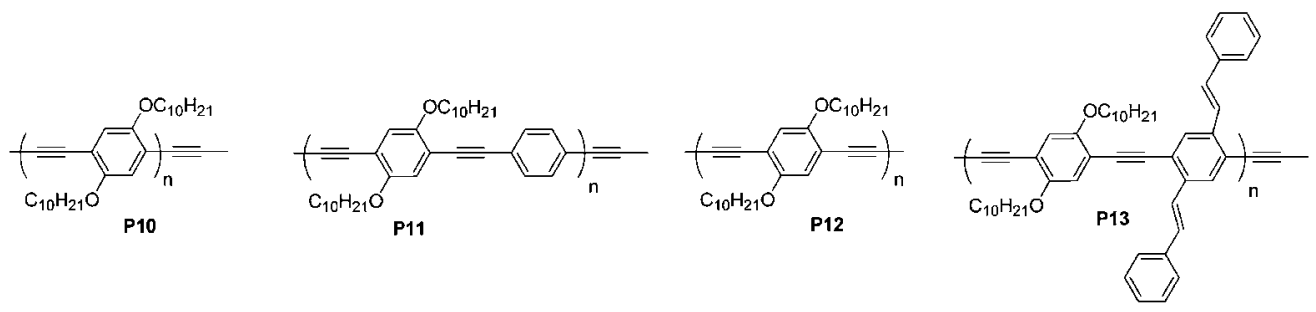

Fig. 5 The structures of four representative linear conjugated poly(phenylenevinylene)s (P10, P11, P12 and P13). 


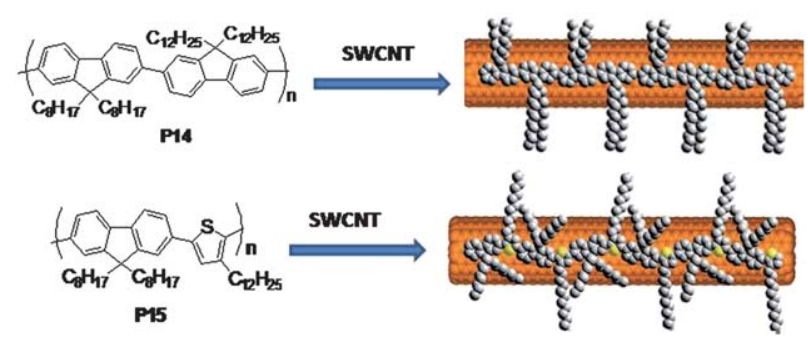

Fig. 6 A cartoon representation of the interaction of poly(9,9-dialkylfluorene) P14 and poly(9,9-dialkylfluorene-co- 3-alkylthiophene) P15 polymers with SWNTs (reproduced with permission from ref. 44. Copyright 2008 American Chemical Society).
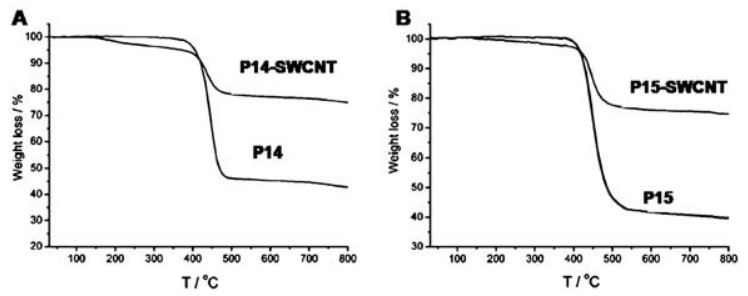

Fig. 7 Thermogravimetric analysis data for polymer P14 (A) and P15 (B), showing thermal decomposition profiles for the free polymer and polymer-nanotube complexes (reproduced with permission from ref. 44 . Copyright 2008 American Chemical Society).

confirm that the polymer chains can disrupt SWNT bundles, resulting in individual nanotubes that are uniformly coated with polymer. Moreover, using four-point-probe measurements, it was found that the conductivity of thin films of polymer-nanotube complexes was only slightly lower than what was observed for films of pristine nanotubes. ${ }^{44}$ The complexes of P14 and P15 with CNTs seem to have promising properties for optoelectronic device applications, however, the applications of these materials are yet to be reported.

\section{The interaction of polythiophenes with CNTs}

Polythiophene derivatives are another important class of materials which are used in many applications such as photovoltaic devices, field effect transistors and sensors. In the fabrication of photovoltaic devices, they have usually been blended with fullerene to achieve optimum charge separation. ${ }^{67}$ However, utilizing CNTs as electron acceptor material instead of fullerene and derivatives has been proved to be highly appealing because of a number of reasons. One of the most important reasons is that a high surface contact area can be achieved by an intimate interaction of PT with CNTs. In the earlier examples, devices fabricated from this composite material suffered from low efficiency. ${ }^{20,21}$ For example, the use of SWCNT presented some problems such as short circuit because of its high metallic contents. However, in the recent works the researchers offered some solutions to circumvent the low efficiency problem, for example, by enriching semiconducting contents of SWCNTs and solubilising them efficiently in polythiophene solution to obtain homogeneous $\mathrm{CNTs} / \mathrm{PT}$ composite material. ${ }^{68}$
Transparent conductive films are quite essential for large area optoelectronic device applications including flat panel displays, electronic papers, sensors, and solar cells. Currently indium tin oxide (ITO)-based transparent conductive films are utilized in the fabrication of these devices, however, they present some technical problems. To this end, easy and inexpensive methods and light-weight, strong and stable materials for the fabrication of transparent conductive films are highly sought after. Swager and co-workers have described a new and convenient method to fabricate free-standing, transparent, and conductive SWCNT films from highly stable dispersion of high molecular weight regiorandom poly(3-hexylthiophene) in organic solvents. These films can be easily transferable onto any substrate such as plastic or silicone oxide wafer and the thickness of the films could be adjusted by simply varying the dispersion concentration. They also demonstrated the use of these materials as an electrode for the electropolymerization of 3-hexylthiophene to give an additional layer of poly(3-hexylthiophene). ${ }^{27}$

Bao and co-workers studied systematically the solubilization of SWCNTs into various organic solvents such as o-dichlorobenzene (DCB), THF and chloroform by using a series of conjugated polymers based on poly(phenylene vinylene and polythiophene with different electronic properties and regioregularities. The highest dispersion concentration achieved was $2.75 \mathrm{mg} \mathrm{mL}^{-1}$ in DCB with regioregular poly(3-hexylthiophene) and poly(3-dodecylthiophene) for SWNTs. DCB was found to be the best solvent for dispersion, followed by chloroform and THF. High concentration dispersion of CNTs in the conjugated polymer solution in organic solvents enabled the authors to observe alignment of CNTs which exhibited lyotropic liquidcrystalline phase. This feature is very remarkable and can be exploited in the fabrication of large area transparent conductive films to construct optoelectronic devices. ${ }^{25}$

The relationship between the morphology of the SWCNT/PT film and the photovoltaic device performance was also investigated. It was demonstrated that photovoltaic devices based on a film made from the composite of $\mathrm{CNTs} / \mathrm{PT}$ with relatively high crystallinity displayed much enhanced performance. The work function of carbon nanotubes modulated by electron transferring from PT to SWCNTs was proposed to explain the high opencircuit voltage obtained from the photovoltaic devices based on the SWCNTs/PT system. ${ }^{26}$

The ability of polythiophene derivatives to solubilise CNTs efficiently to form homogeneous solution has been taken as advantage and utilized in chemiresistive sensor fabrication using spin-coating technique. Adding different functionalities to the polythiophene backbone, it was possible to detect a number of different analytes such as chemical warfare agents ${ }^{69}$ and to distinguish the isomers of xylene. ${ }^{70}$

\section{Block copolymers as a dispersant agent}

Although conjugated polymers used in the previous section solubilise and stabilize the CNTs through the formation of strong $\pi-\pi$ stack interactions, the amount of CNTs could be solubilised is rather limited and the stability of the resulting dispersions may not be so high because the polymers posses inherently large hydrophobic conjugated backbone which limits the overall solubility. In order to increase the solubility of CNTs 
in CPNs solution, one can either attach large solubilising groups to the polymer backbone as side chains or copolymerize conjugated polymers with non-conjugated well-soluble polymers in organic solvents.

To this end, recently conjugated block copolymers have been reported as a new class of efficient dispersant agents for CNTs. ${ }^{45-47}$ The polymers were designed in such a way that one block of the polymer composed of a conjugated polymer that would interact with the carbon nanotube walls via $\pi-\pi$ interaction, while the other block(s) will impart solubility to the debundled nanotubes by forming a steric barrier or repulsive interaction between polymer-wrapped nanotubes. Using these polymers it would also be possible to prepare good quality, uniform composite films for device applications.

For this purpose, Zou and co-workers first synthesized a conjugated block copolymer made from 3-hexylthiophene and styrene (P16) (Fig. 8) and investigated its ability to disperse CNTs in various solvents. Both SWCNTs and MWCNTs were well dispersed in chloroform, toluene and THF in the presence of P16 with gentle sonication. To achieve a good dispersion, the minimum mass ratio of $\mathbf{P 1 6}$ to CNTs claimed to be 0.6 and 0.5 for SWCNT and MWCNT, respectively. Moreover, the highest CNT concentration of stable CNT dispersions was found to be around $2.5 \mathrm{mg} \mathrm{mL}^{-1}$ and $3.0 \mathrm{mg} \mathrm{mL}^{-1}$ for SWCNT (using $2: 1$ polymer to SWCNTs mass ratio) and MWCNT (using $1: 1$ polymer to MWCNTs mass ratio), respectively. The as-dispersed CNTs are very stable, no precipitation was found even after one year of standing at room temperature. They also investigated the interaction between P16 and SWCNT and MWCNTs using various spectroscopic techniques such as ${ }^{1} \mathrm{H}-\mathrm{NMR}$, UV-Vis, Fluorescence, Raman as well as by TEM. Furthermore, the electrical conductivity of the film of CNTs/P16 composite was measured using a four-probe method. ${ }^{46}$

Fluorescence emission measurements exhibited that after dispersing SWCNTs in the P16 block copolymer solution, 97\% of the fluorescence emission was quenched indicating a strong molecular interaction between conjugated block copolymers and CNTs while poly(3-hexylthiophene)/SWCNTs dispersion showed that only $75 \%$ of the fluorescence emission was quenched, suggesting there is a much stronger interaction between P16 and CNTs than that between poly(3-hexylthiophene) and CNTs (Fig. 8).

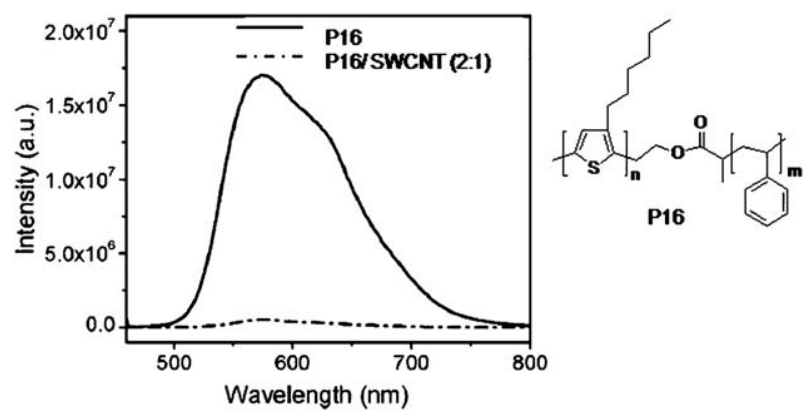

Fig. 8 The fluorescence spectra of pure of poly(3-hexylthiophene)- $b$ polystyrene (P16) and P16/SWCNTs (2:1) dispersion in chloroform (reproduced with permission from ref. 46. Copyright 2008 Wiley-VCH Verlag GmbH \& Co. KGaA).
The same group in 2009 designed a series of block copolymers in which one block is composed of poly(3-hexylthiophene) whereas the other block is a hydrophilic polymer that can dissolve in polar solvents. They demonstrated that the composite of CNTs with these conjugated block copolymers could be made dispersible in various solvents including chloroform, toluene, methanol, ethanol, DMF, and acetonitrile by tuning the structure of the non-conjugated blocks of the conjugated polymers. ${ }^{47}$ To be able to dissolve the CPs/CNTs composites in a number of different solvents could be a very useful feature in the device fabrication for building up layers with different functions on top of each other without dissolving the previous layer.

\section{Conjugated polymers used as a dispersant agent in aqueous media}

Dispersing CNTs in aqueous media is quite an important endeavour because of a number of reasons:

For the optoelectronic device applications, water solubility of CNT/CP composites enables their processing under environmentally friendly conditions. It could also be possible to selfassemble these materials through electrostatic interactions (i.e., layer-by-layer assembly) using polyelectrolyte conjugated polymers to obtain more complex structures and films with defined thickness and functionalities. Furthermore, the water dispersible $\mathrm{CP} / \mathrm{CNT}$ composite materials are particularly important for biomedical, chemo- and bio-sensor applications. Several recent reports have shown that CNTs are able to assist in the killing of cancer cells upon irradiation because of the near IR absorption property of CNTs, and that CNTs can also deliver therapeutic agents such as plasmid DNA, siRNA, or anticancer drugs to cells, in a specific manner. ${ }^{48-50}$

Adronov and co-workers reported an interesting and inexpensive approach for the fabrication of conductive nanotube patterns that can be suitable for large-area electronic devices. For this, they first prepared complexes of single-walled carbon nanotubes and two anionic conjugated polyelectrolytes, P17 and P18 (Fig. 9). The complex formation between the polymers and CNTs were confirmed by using spectroscopic techniques such as UV-Vis, fluorescence, Raman, and electron microscopy. The patterning of these complexes were achieved through electrostatic attraction with a prepatterned cationically charged surface. The electrical properties of the pattern films were investigated and they were found to be electrically conducting. ${ }^{51}$ Although this approach is quite promising, further improvements are required before the device fabrication.

Kimura and co-workers have demonstrated a technique of preparing transparent conductive films composed of SWNTs and water-soluble conjugated polymers. The films were prepared by a layer-by-layer (LBL) assembly of anionic P19-A/SWNTs and cationic P19-C (Fig. 9) on various substrates such as quartz substrates, gold surface of quartz crystal microbalances (QCMs), and interdigitated microcircuit electrodes (IMEs). The formation of LBL films containing SWNTs was monitored by UV-vis, fluorescence spectra and frequency changes of the quartz crystal microbalances. It was shown that the electric conductivity within the LBL films can be controlled by the deposition direction in the LBL formation. ${ }^{52}$ 


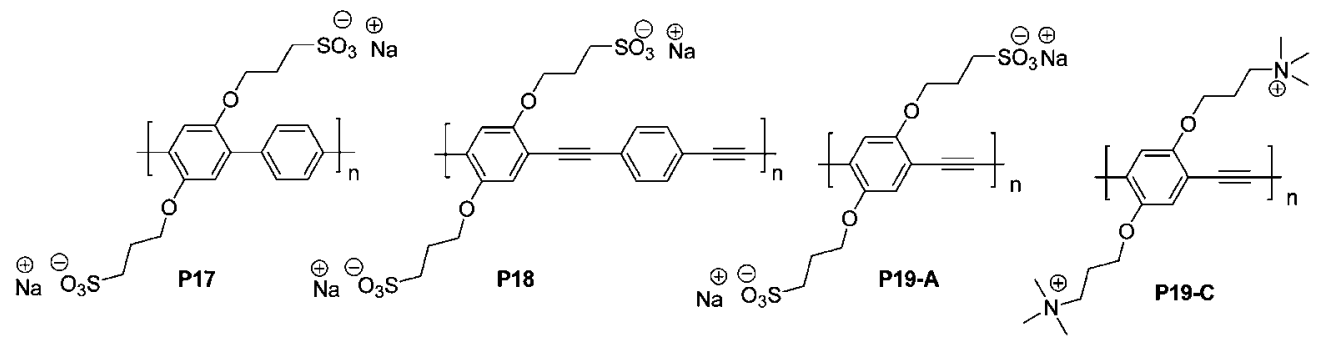

Fig. 9 The structures of poly[2,5-bis(3-sulfonatopropoxy)-1,4-phenylene-alt-1,4-phenylene) sodium salt (P17), poly[2,5-bis(3-sulfonatopropoxy)-1,4ethynylphenylene-alt-1,4-ethynylphenylene $]$ sodium salt (P18), poly[ $p$ - $\{2,5$-bis(3-propoxysulfonic acidsodiumsalt) $\}$ phenylene $]$ ethynylene (P19-A) and poly $[p$ - $\{2,5$-bis(3-propoxytrimethylammonium $\}$ phenylene $]$ ethynylene (P19-C).

Electrophoretic deposition (EPD) method was adopted by Adronov and co-workers in the construction of good quality and uniform films of $\mathrm{CPs} / \mathrm{CNTs}$ composites. In this technique, basically the charged particles or polymers in a stable suspension migrate toward an electrode under the influence of an electric field and deposited onto an electrode. For this purpose, they synthesized a conjugated tertiary amine-functionalized polymer, (P20) (Fig. 10). The formation of stable solutions in organic solvents, as well as in water upon protonation of the amine groups, indicates strong supramolecular interactions between the polymers and the carbon nanotube surface. UV-vis absorption spectroscopy and Raman spectroscopy were utilized to characterize the resulting functionalized nanotubes. The pure polymer P20 and P20/SWNT composite were deposited on the various substrates including ITO using EPD method. The resulting films were characterized by SEM showing uniform coatings that are free of cracks. ${ }^{53}$ This method is quite promising in the fabrication of CPs/CNTs films for the active layers of optoelectronic devices because the film quality and thicknesses could be controllable; furthermore it is a cheap, easy and versatile method.

Recently amphiphilic, linear conjugated polymer, P19-A has been utilized to dissolve SWCNTs very efficiently in aqueous media and almost $80 \%$ of the solubilised tubes were found to be individualized. Although P19-A has a linear and rigid structure, self-assembled superstructures were observed to form in which a polymer monolayer helically wraps the nanotube surface. The formation of these helically wrapped-superstructures were confirmed by Vis-NIR absorption spectroscopy, AFM and TEM as well as via molecular dynamics (MD) simulations. ${ }^{54}$ Fig. 11
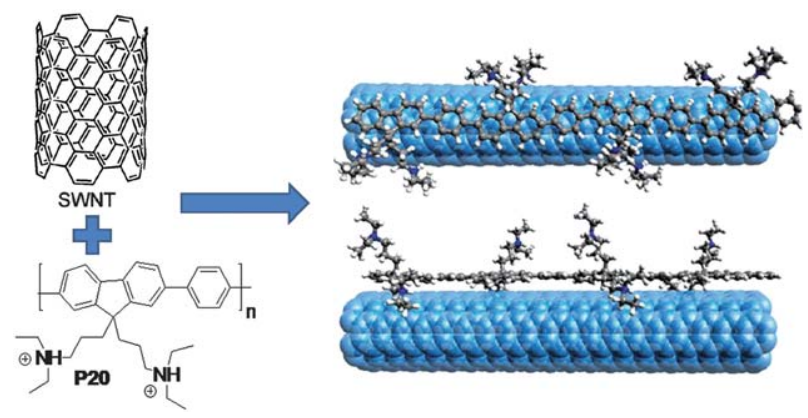

Fig. 10 Schematic representation of the supramolecular interaction between protonated poly(9,9-bis(diethylaminopropyl)-2,7-fluoreneco1,4-phenylene) (P20) and SWNTs, showing a top and side view on the upper and lower right, respectively. (reproduced with permission from ref. 53. Copyright 2010 American Chemical Society). shows some snapshots of the interaction process between a SWNT and P19-A (containing 20 repeating units) obtained through MD simulation obtained over a $1-20 \mathrm{~ns}$ period as well as a TEM image of a P19-A/SWNT complex.

Very recently, we reported that the CNTs could be made dispersible in the aqueous dispersion of conjugated polymer nanoparticles composed of hydrophobic conjugated polymers. The attachments of conjugated polymer nanoparticles (CPNs) made from P21 (Fig. 13) were confirmed by several techniques including spectroscopic (UV-Vis, Fluorescence, and Raman) and microscopic (SEM and TEM) techniques. The optical properties of the conjugated polymer nanoparticles were not substantially affected by the CNTs. ${ }^{55}$ After dispersing the CNTs using 40 and $70 \mathrm{~nm}$-sized CPN-water dispersions, $25 \%$ and $10 \%$ quenching in the fluorescent emission of the CPs was observed, respectively. This feature can be very useful for biological applications such as bioimaging and biosensing which requires direct fluorescent labelling of CNTs that has usually been hindered by strong light emission quenching characteristic of CNTs. Furthermore, therapeutic agents such as plasmid DNA, siRNA, or anticancer drugs can be attached to nanoparticles/CNT complexes and delivered to cells in a specific manner. Fig. 12 shows the scanning electron microscopy (SEM) and CPN/CNT water dispersion, under ambient and UV-light irradiation.

It was demonstrated quite recently that it is possible to disperse SWCNTs in multiple organic solvents by using a water soluble conjugated polyelectrolyte as a dispersant in the presence of a phase transfer catalyst (PTC) such as 18 -crown- $6 .{ }^{56}$

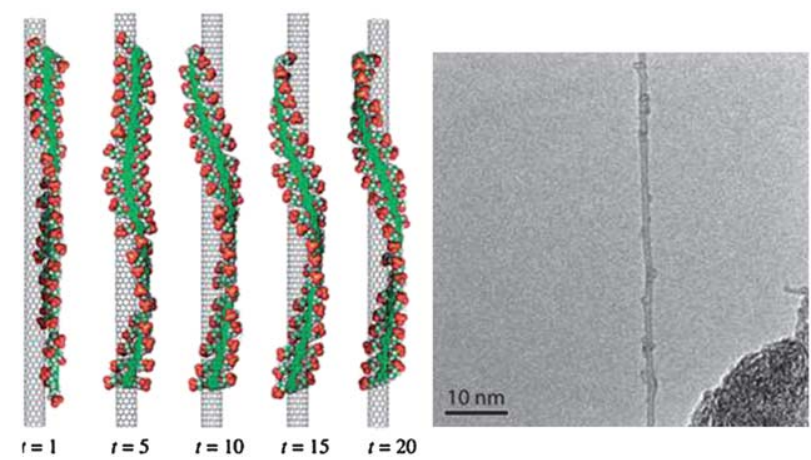

Fig. 11 Snapshots of the interaction process between a SWNT and P19A (containing 20 repeating units) obtained through MD simulation obtained over a 1-20 ns period. TEM image of a P19-A/SWNT (reproduced with permission from ref. 54. Copyright 2009 American Chemical Society). 

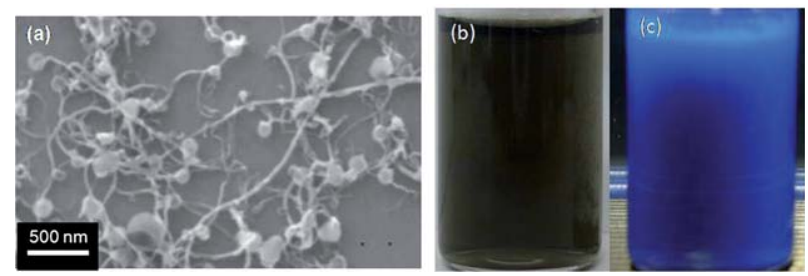

Fig. 12 (a) CPNs attached to CNTs imaged by SEM, (b, c) CPN/CNT water dispersion, under ambient and UV-light irradiation respectively (reproduced with permission from ref. 55. Copyright 2010 Royal Society of Chemistry).

\section{Spectroscopic and computational studies to reveal the interactions between $\mathrm{CNT}$ and $\mathrm{CP}$}

It is important to reveal the exact nature of the interaction between CNTs and CPs for fundamental studies as well as the optoelectronic device applications of CNT/CP composites. It is proposed by many groups that the energy is transferred from CPs to CNTs. Nicholas and coworkers studied the energy transfer from the conjugated polymers P22 and P23 (see Fig. 13) to CNTs by providing a direct evidence using photoluminescence excitation mapping. The energy was transferred from polymers to SWCNTs when the polymers were excited across their minimum energy gaps. ${ }^{57}$

Following on, they went on and conducted a detailed work to reveal the polymer structure and solvent effects on the selective dispersion of single-walled carbon nanotubes. They investigated the dispersibility of SWCNTs in a series of fluorene-based polymers using various solvents and subsequently characterized the resulting dispersions by optical absorbance, photoluminescence-excitation mapping and electron microscopic imaging as well as by computer modeling. Their studies confirmed that both the polymer structure and solvent used strongly influenced the dispersion of the nanotubes, leading in some cases to very high selectivity in terms of diameter and chiral angle. They observed highest selectivities using toluene with the rigid polymers P26 and P22 (Fig. 13) to suspend isolated nanotubes and concluded that the specific nanotube species selected were also dependent on the solvent used and could be adjusted by the use of THF or xylene. On the other hand, the polymers were shown to be less selective having a structure with flexible conformations, but show an enhanced overall solubilization of CNTs. The nanotubes were suspended as bundles rather than as isolated tubes, which lead to a quenching of their photoluminescence when chloroform was used as the solvent, however, a large increase in the overall solubilization was observed. ${ }^{58}$

In 2009, $\mathrm{Li}$ and co-workers reported their findings on the energy transfer from fluorene-based polymers, P23 and P24 (Fig. 13) to single-walled carbon nanotubes (SWNTs). In this study, they explored the photon conversion process between aromatic polymers and SWNTs and suggested a convenient method of adjusting the desired wavelength for the optimal energy conversion. The energy transfer process was investigated by photoluminescence excitation (PLE) and fluorescence lifetime measurements. It was demonstrated that the excitation wavelength could be tune in a wide wavelength range from 388 to 480 $\mathrm{nm}$ depending on the concentration of excess P25 (P26) polymers. The authors utilized the PLE mapping technique to monitor the energy transfer process and to study the SWNT binding competition between P23 and P24 by concluding that SWNTs were preferentially bound with $\mathbf{P 2 4}$ polymers; this was also supported by the molecular dynamic simulation studies. ${ }^{59}$

Collison and co-workers reported the physical interaction of SWCNTs with a conjugated polymer, $\mathbf{P 2 2}$ on the basis of the spectroscopic assignments of various conformational species of different optical signature in $N, N$-dimethylacetamide (DMA) dispersions. They showed that energy transfer from nonaggregated P22 leads to enhanced SWCNT fluorescence in solutions of poorer solvent quality. Energy transfer from polymer chain lengths that are torsionally restricted is not observed. They interpreted these conclusions as that any electron transfer taking place is occurring through a concerted Dexter mechanism and that use of SWCNTs as an electron acceptor will likely have associated drawbacks. ${ }^{60}$

Poly(3-hexylthiophene) (P3HT)/SWNTs composites were prepared using a series of SWNTs with various defect contents on their surfaces. UV-visible and photoluminescence (PL) spectra showed that the carbon nanotubes quench the PL of $\mathrm{P} 3 \mathrm{HT}$ in the composite, indicating that electron transfer occurs from photo-excited P3HT to the SWNTs. Interestingly, it was observed that the electron transfer from P3HT to carbon nanotubes was disrupted by the presence of defects on the SWNT surfaces. However, the PL lifetime of P3HT in the composites

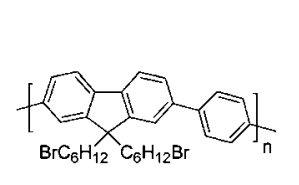

P21

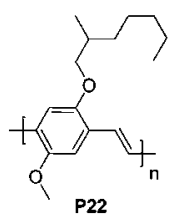

P22

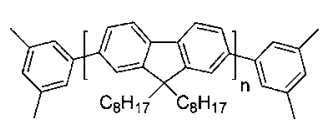

P23

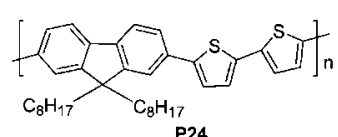

P24

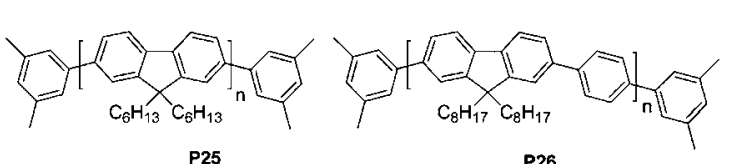

P25
P26

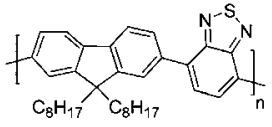

P27

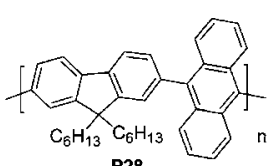

P28

Fig. 13 The chemical structures of poly[9,9-bis-(6-bromohexylfluorene-2,7-diyl)-co-(benzene-1,4-diyl)] (P21), poly[2'-methoxy-5-(2'-ethylhexyloxy)1,4-phenylenevinylene] (P22), poly[9,9-dioctylfluorenyl-2,7-diyl] (P23), poly[(9,9-dioctylfluorenyl-2,7-diyl)-co-(bithiophene)] (P24), poly[9,9-dihexylfluorenyl-2,7-diyl] (P25), poly[(9,9-dioctylfluorenyl-2,7-diyl)-co-(1,4-phenylene)] (P26) and poly[(9,9-dioctylfluorenyl-2,7-diyl)-alt-co-(1,4-benzo-2,10,3thiadiazole)] (P27), poly[(9,9-dihexylfluorenyl-2,7-diyl)-co-antracene] (P28). 
was found to be the same as that of pure P3HT in solution, indicating the formation of a ground-state non-fluorescent complex of P3HT/SWNTs. ${ }^{61}$

Polymer-wrapped single-walled carbon nanotubes were prepared with two different conjugated polymers showing different affinity for SWCNTs. These hybrid systems were used to obtain a sample enriched of semiconducting SWNTs and measure their intrinsic lifetime that is found to be in the range of 30-40 ps. In the SWNTs dispersion with P23, the fluorescence of the polymer is quenched due to the energy transfer towards the nanotube. Conversely, in the case of P22 the PL decay became slower indicating the formation of a more delocalized excited state in the composite. The quenching of the $\mathbf{P 2 2}$ photoluminescence is observed when the polymer is in contact with bundles of SWNTs owing to the presence of metallic nanotubes. ${ }^{62}$

In order to further the understanding of interaction nature between CNTs and CPs, molecular dynamics simulations were performed on the interactions between SWCNTs and a series of conjugated polymers including polythiophene (PT), polypyrrole (PP), P3, and P1 by computing the intermolecular interaction energy between SWCNTs and polymer molecules. The morphology of polymers physisorbed to the surface of nanotubes was studied by the radius of gyration $(R g)$ and the alignment angle by taking into account the influence of nanotube radius and temperature on the interfacial adhesion of nanotube-polymer and $R \mathrm{~g}$ of polymers. The simulation results showed that the intermolecular interaction was strongly influenced by the nature of monomers and nanotube radius, but the influence of temperature could be negligible. Accordingly, the strength of the binding between the SWCNTs and CPs was found to be in the following decreasing order of PT, PPy, P1 and $\mathbf{P 3}$ indicating that the heterocyclic conjugated polymers can interact efficiently with CNTs. ${ }^{63}$

\section{Extraction of Single Species of Single-Walled Carbon Nanotubes by fluorene-based polymers}

In a SWCNT mixture, metallic and semi-conducting tubes with various sizes coexist and having a mixture of different kinds of species of SWCNTs is not desirable for fundamental research as well as in the use of high-performance device fabrications. It is therefore highly sought-after to develop purification methods to obtain single species of SWCNTs. There are many approaches to achieve this goal. ${ }^{64}$ Among them, recently a method involves the use of conjugated polymers as a dispersant has received considerable attentions. This method is based on the ability of specific conjugated polymers to efficiently disperse certain nanotube species with a high degree of selectivity. Nicholas and co-workers demonstrated the potential of fluorene-based polymers such as P23, P25, P26 and P27 (the structures shown in Fig. 13) to selectively solubilize certain nanotube species. Even small changes in the polymer structure lead to selection on the basis of both chiral angle and nanotube diameter. P23 has shown particularly strong selectivity and leads to much improved optical spectra. Computer simulations of the interaction of P23 with SWNTs of various diameters give some insight into the mechanism that causes the selectivity, but ultimately show the system to be more complex than can be currently modelled. ${ }^{65}$
$\mathrm{Li}$ and co-workers also reported around the same time the ability of fluorene-based polymers to selectively wrap SWNTs with certain chiral angles or diameters depending on their chemical structures. They demonstrated that high purity of SWNTs (appx. $79 \%$ of the semiconducting SWNT ensemble) could be obtained by polymer-assisted extraction from the mixture of narrow diameter distributed SWNTs. ${ }^{66}$

\section{Conclusions}

Carbon nanotubes are very exciting materials with interesting properties and having a number of potential applications in various areas spanning from optoelectronics to biotechnology. However, their solubility problem limits their wide accessibility for many applications. Their insolubility stems from the bundling of individual carbon nanotubes caused by weak intermolecular non-covalent interactions-mainly van der Waals interactions. To this end, many approaches have been pursued to circumvent this problem including non-covalent and covalent functionalization of CNT surfaces. While the covalent modification may alter the intrinsic properties of carbon nanotubes, the non-covalent modification keeps untouched to those properties.

Among many non-covalent functionalization, conjugated polymer-based functionalization of CNTs are very appealing because of a number of reasons. The films and nanocomposites made from well-dispersed CNTs in conjugated polymer solutions can find many applications in device fabrications including light emitting diodes, solar cells, transparent conductive films and sensors because the composite materials of CPs/CNTs combine the good thermal-/photostability, high mechanical strength, and high electrical conductivity of carbon nanotubes with excellent optoelectronic properties of conjugated polymers.

Especially, easy and inexpensive methods and light-weight, flexible, strong and stable materials for the fabrication of transparent conductive films are quite desirable for large area optoelectronic device applications including flat panel displays, electronic papers, sensors, and solar cells. Currently indium tin oxide (ITO)-based transparent conductive films are used in the fabrication of these devices, however, ITO-based materials present a number of technical problems and replacement of ITO with more suitable transparent conductive films are required to advance the field. Some works have already been reported in the use CP/CNT-based composite materials for the fabrication of transparent conductive films indicating that these materials are very promising; however, more works should be conducted to improve the film quality and methods used.

The water dispersible CP/CNT composite materials could also be very useful for biomedical, chemo- and bio-sensor applications. The conjugated polymers could solubilise CNTs efficiently in water and act as fluorescent label if we could prevent CNTs to quench completely the fluorescent emission of CPs. Furthermore, therapeutic agents such as plasmid DNA, siRNA, or anticancer drugs can be attached to $\mathrm{CP} / \mathrm{CNT}$ complexes and delivered to cells in a specific manner and fluorescent labelling will provide us with information on the whereabouts of the CNTs. However, to the our best of knowledge there is no work in this area involving the use of $\mathrm{CP} / \mathrm{CNT}$ water dispersion to demonstrate the aforementioned approach; thus, this area seems to be full of promising and awaiting to be discovered. 


\section{References}

1 (a) S. Iijima, Nature, 1991, 354, 56; (b) P. M. Ajayan and S. Iijima, Nature, 1993, 361, 334.

2 Carbon Nanotubes and Related Structures, ed. D. Guldiand N. Martin, Wiley-VCH, 2010.

3 P. M. Ajayan, Chem. Rev., 1999, 99, 1787.

4 R. H. Baughman, A. A. Zakhidov and W. A. de Heer, Science, 2002, 297, 787.

5 M. in het Panhuis, J. Mater. Chem., 2006, 16, 3598.

6 H.-C. Wu, X. Chang, L. Liu, F. Zhaoa and Y. Zhao, J. Mater. Chem., 2010, 20, 1036

7 M. Bansal, R. Srivastava, C. Lal, M. N. Kamalasananb and L. S. Tanwara, Nanoscale, 2009, 1, 317.

8 J. L. Delgado, P.-A. Bouit, S. Filippone, M. A. Herranza and N. Martin, Chem. Commun., 2010, 46, 4853.

9 D. Tasis, N. Tagmatarchis, A. Bianco and M. Prato, Chem. Rev., 2006, 106, 1105.

10 P. Singh, S. Campidelli, S. Giordani, D. Bonifazi, A. Bianco and M. Prato, Chem. Soc. Rev., 2009, 38, 2214.

11 K. Balasubramanian and M. Burghard, Small, 2005, 1, 180.

12 M. T. Byrne and Y. K. Gun'ko, Adv. Mater., 2010, 22, 1672.

13 M. Baibarac and P. Gómez-Romero, J. Nanosci. Nanotech., 2006, 6, $1-14$.

14 Y.-L. Zhao and J. F. Stoddart, Acc. Chem. Res., 2009, 42, 1161.

15 V. C. Moore, M. S. Strano, E. H. Haroz, R. H. Hauge, R. E. Smalley, J. Schmidt and Y. Talmon, Nano Lett., 2003, 3, 1379.

16 A. Nish and R. J. Nicholas, Phys. Chem. Chem. Phys., 2006, 8, 3547

17 J. G. Duque, A. N. G. Parra-Vasquez, N. Behabtu, M. J. Green, O. E. A. L. Higginbotham, B. K. Price, A. D. Leonard, H. K. Schmidt, B. Lounis, J. M. Tour, S. K. Doorn, L. Cognet and M. Pasquali, ACS Nano, 2010, 4, 3063.

18 R. Haggenmueller, S. S. Rahatekar, J. A. Fagan, J. Chun, M. L. Becker, R. R. Naik, T. Krauss, L. Carlson, J. F. Kadla, P. C. Trulove, D. F. Fox, H. C. DeLong, Z. Fang, S. O. Kelley and J. W. Gilman, Langmuir, 2008, 24, 5070.

19 S. Park, H.-S. Yang, D. Kim, K. Jo and S. Jon, Chem. Commun., 2008, 2876.

20 S. A. Curran, P. M. Ajayan, W. J. Blau, D. L. Carroll, J. N. Coleman, A. B. Dalton, A. P. Davey, A. Drury, B. McCarthy, S. Maier and A. Strevens, Adv. Mater., 1998, 10, 1091.

21 (a) E. Kymakis and G. A. J. Amaratunga, Appl. Phys. Lett., 2002, 80, 112; (b) E. Kymakis, I. Alexandrou and G. A. J. Amaratunga, J. Appl. Phys., 2003, 93, 1764.

22 S. L. Hellstrom, H. W. Lee and Zhenan Bao, ACS Nano, 2009, 3, 1423.

23 H. Zhao, W. Z. Yuan, L. Tang, J. Z. Sun, H. Xu, A. Qin, Y. Mao, J. K. Jin and B. Z. Tang, Macromolecules, 2008, 41, 8566.

24 D. R. Kauffman and A. Star, Angew. Chem., Int. Ed., 2008, 47, 6550.

25 H. W. Lee, W. You, S. Barman, S. Hellstrom, M. C. LeMieux, J. H. Oh, S. Liu, T. Fujiwara, W. M. Wang, B. Chen, Y. W. Jin, J. M. Kim and Z. Bao, Small, 2009, 5, 1019.

26 J. Geng and T. Zeng, J. Am. Chem. Soc., 2006, 128, 16827.

27 H. Gu and T. M. Swager, Adv. Mater., 2008, 20, 4433.

28 H. Ago, K. Petritsch, M. S. P. Shaffer, A. H. Windle and R. H. Friend, Adv. Mater., 1999, 11, 1281.

29 S. Curran, A. P. Davey, J. N. Coleman, A. B. Dalton, B. McCarthy, S. Maier, A. Drury, D. Gray, M. Brennan, K. Ryder, M. Lamy de la Chapelle, C. NJournet, P. Bernier, H. J. Byrne, D. Carroll, P. M. Ajayan, S. Lefrant and W. J. Blau, Synth. Met., 1999, 103, 2559.

30 A. B. Dalton, C. Stephan, J. N. Coleman, B. McCarthy, P. M. Ajayan, S. Lefrant, P. Bernier, W. J. Blau and H. J. Byrne, J. Phys. Chem. B, 2000, 104, 10012.

31 J. N. Coleman, A. B. Dalton, S. Curran, A. Rubio, A. P. Davey, A. Drury, B. McCarthy, B. Lahr, P. M. Ajayan, S. Roth, R. C. Barklie and W. J. Blau, Adv. Mater., 2000, 12, 213.

32 B. McCarthy, J. N. Coleman, R. Czerw, A. B. Dalton, D. L. Carroll and W. J. Blau, Synth. Met., 2001, 121, 1225.

33 A. Star, J. F. Stoddart, D. Steuerman, M. Diehl, A. Boukai, E. W. Wong, X. Yang, S.-W. Chung, H. Choi and J. R. Heath, Angew. Chem., Int. Ed., 2001, 40, 1721.

34 A. Star and J. F. Stoddart, Macromolecules, 2002, 35, 7516.

35 D. W. Steuerman, A. Star, R. Narizzano, H. Choi, R. S. Ries, C. Nicolini, J. F. Stoddart and J. R. Heath, J. Phys. Chem. B, 2002, 106, 3124 .
36 A. Star, Y. Liu, K. Grant, L. Ridvan, J. F. Stoddart, D. W. Steuerman, M. R. Diehl, A. Boukai and J. R. Heath, Macromolecules, 2003, 36, 553.

37 Wenhui, A. Malkovskiy, Q. Chu, A. P. Sokolov, M. L. Colon, M. Meador and Y. Pang, J. Phys. Chem. B, 2008, 112, 12263.

38 M. Kimura, N. Miki, N. Adachi, Y. Tatewaki, K. Ohtaa and H. Shiraiab, J. Mater. Chem., 2009, 19, 1086.

39 W. Yi, A. Malkovskiy, Y. Xu, X.-Q. Wang, A. P. Sokolov, M. Lebron-Colon, M. A. Meador and Y. Pang, Polymer, 2010, 51, 475.

40 W. Z. Yuan, J. Z. Sun, Y. Dong, M. Halussler, F. Yang, H. P. Xu, A. Qin, J. W. Y. Lam, Q. Zheng and B. Z. Tang, Macromolecules, 2006, 39, 8011.

41 J. Chen, H. Liu, W. A. Weimer, M. D. Halls, D. H. Waldeck and G. C. Walker, J. Am. Chem. Soc., 2002, 124, 9034.

42 J. Chen, R. Ramasubramaniam, C. Xue and H. Liu, Adv. Funct. Mater., 2006, 16, 114.

43 N. A. Rice, K. Soper, N. Zhou, E. Merschrod and Y. Zhao, Chem. Commun., 2006, 4937.

44 F. Cheng, P. Imin, C. Maunders, G. Botton and A. Adronov, Macromolecules, 2008, 41, 2304.

45 E. Nativ-Roth, R. Shvartzman-Cohen, C. Bounioux, M. Florent, D. Zhang, I. Szleifer and R. Yerushalmi-Rozen, Macromolecules, 2007, 40, 3676.

46 J. Zou, L. Liu, H. Chen, S. I. Khondaker, R. D. McCullough, Q. Huo and L. Zhai, Adv. Mater., 2008, 20, 2055.

47 J. Zou, S. I. Khondaker, Q. Huo and L. Zhai, Adv. Funct. Mater., 2009, 19, 479-483.

48 B. C. Satishkumar, L. O. Brown, Y. Gao, C.-C. Wang, H.-L. Wang and S. K. Doorn, Nat. Nanotechnol., 2007, 2, 560.

49 K. Kostarelos, A. Bianco and M. Prato, Nat. Nanotechnol., 2009, 4, 627

50 N. W. S. Kam, M. O'Connell, J. A. Wisdom and H. Dai, Proc. Natl. Acad. Sci. U. S. A., 2005, 102, 11600.

51 F. Cheng, P. Imin, S. Lazar, G. A. Botton, G. de Silveira, O. Marinov, J. Deen and A. Adronov, Macromolecules, 2008, 41, 9869.

52 N. Adachi, T. Fukawa, Y. Tatewaki, H. Shirai and M. Kimura, Macromol. Rapid Commun., 2008, 29, 1877.

53 T. Casagrande, P. Imin, F. Cheng, G. A. Botton, I. Zhitomirsky and A. Adronov, Chem. Mater., 2010, 22, 2741.

54 Y. K. Kang, O.-S. Lee, P. Deria, S. H. Kim, T.-H. Park, D. A. Bonnell, J. G. Saven and M. J. Therien, Nano Lett., 2009, 9, 1414.

55 B. Baykal, V. Ibrahimova, G. Er, E. Bengu and D. Tuncel, Chem. Commun., 2010, 46, 6762.

56 P. Deria, L. E. Sinks, T.-H. Park, D. M. Tomezsko, M. J. Brukman, D. A. Bonnell and M. J. Therien, Nano Lett., 2010, 10, 4192.

57 A. Nish, J.-Y. Hwang, J. Doig and R. J. Nicholas, Nanotechnology, 2008, 19, 095603

58 J.-Y. Hwang, A. Nish, J. Doig, S. Douven, C.-W. Chen, L.-C. Chen and R. J. Nicholas, J. Am. Chem. Soc., 2008, 130, 3543.

59 F. M. Chen, W. Zhang, M. Jia, L. Wei, X.-F. Fan, J.-L. Kuo, Y. Chen, M. B. Chan-Park, A. Xia and L.-J. Li, J. Phys. Chem. C, 2009, 113, 14946.

60 C. J. Collison, S. Pellizzeri and F. Ambrosio, J. Phys. Chem. B, 2009, 113, 5809.

61 J. Geng, B.-S. Kong, S. B. Yang, S. C. Youn, S. Park, T. Joo and H.-T. Jung, Adv. Funct. Mater., 2008, 18, 2659.

62 J. Gao and M. A. Loi, Eur. Phys. J. B, 2009, 75, 121.

63 M. Foroutan and A. T. Nasrabadi, J. Phys. Chem. B, 2010, 114, 5320.

64 C.-H. Liu and H.-L. Zhang, Nanoscale, 2010, 2, 1919.

65 A. Nish, J.-Y. Hwang, J. Doig and R. J. Nicholas, Nat. Nanotechnol., $2007,2,640$.

66 F. Chen, B. Wang, Y. Chen and L.-J. Li, Nano Lett., 2007, 7, 3013.

67 G. Yu, J. Gao, J. C. Hummelen, F. Wudl and A. J. Heeger, Science, 1995, 270, 1789.

68 J. M. Holt, A. J. Ferguson, N. Kopidakis, B. A. Larsen, J. Bult, G. Rumbles and J. L. Blackburn, Nano Lett., 2010, 10, 4627.

69 F. Wang, H. Gu and T. M. Swager, J. Am. Chem. Soc., 2008, 130, 5392.

70 F. Wang, Y. Yang and T. M. Swager, Angew. Chem., Int. Ed., 2008, 47, 8394 . 